\title{
Three-Dimensional Charge Density Wave and Surface-Dependent Vortex-Core States in a Kagome Superconductor $\mathrm{CsV}_{3} \mathrm{Sb}_{5}$
}

\author{
Zuowei Liang, ${ }^{1, \S}$ Xingyuan Hou $\odot,{ }^{2, \S}$ Fan Zhang, ${ }^{3,2, \S}$ Wanru Ma, ${ }^{1}$ Ping Wu, ${ }^{1}$ Zongyuan Zhang, ${ }^{2}$ Fanghang Yu, ${ }^{1}$ \\ J.-J. Ying, ${ }^{1}$ Kun Jiang, ${ }^{3}$ Lei Shan $\odot,{ }^{2,4, *}$ Zhenyu Wang $\odot,{ }^{1, \dagger}$ and X.-H. Chen ${ }^{1,5,6, \$}$ \\ ${ }^{1}$ Department of Physics and Chinese Academy of Sciences Key laboratory of Strongly-coupled Quantum \\ Matter Physics, University of Science and Technology of China, Hefei, Anhui 230026, China \\ ${ }^{2}$ Information Materials and Intelligent Sensing Laboratory of Anhui Province, \\ Institutes of Physical Science and Information Technology, Anhui University, Hefei 230601, China \\ ${ }^{3}$ Beijing National Laboratory for Condensed Matter Physics, and Institute of Physics, \\ Chinese Academy of Sciences, Beijing 100190, China \\ ${ }^{4}$ Key Laboratory of Structure and Functional Regulation of Hybrid Materials of Ministry of Education, \\ Anhui University, Hefei 230601, China \\ ${ }^{5}$ CAS Center for Excellence in Quantum Information and Quantum Physics, Hefei, Anhui 230026, China \\ ${ }^{6}$ Collaborative Innovation Center of Advanced Microstructures, Nanjing 210093, China
}

(Received 8 March 2021; revised 12 May 2021; accepted 7 June 2021; published 2 August 2021)

\begin{abstract}
The transition-metal-based kagome metals provide a versatile platform for correlated topological phases hosting various electronic instabilities. While superconductivity is rare in layered kagome compounds, its interplay with nontrivial topology could offer an engaging space to realize exotic excitations of quasiparticles. Here, we use scanning tunneling microscopy to study a newly discovered $Z_{2}$ topological kagome metal $\mathrm{CsV}_{3} \mathrm{Sb}_{5}$ with a superconducting ground state. We observe charge modulation associated with the opening of an energy gap near the Fermi level. When across single-unit-cell surface step edges, the intensity of this charge modulation exhibits a $\pi$-phase shift, suggesting a three-dimensional $2 \times 2 \times 2$ charge density wave ordering. Interestingly, while conventional Caroli-de Gennes-Matricon bound states are observed inside the superconducting vortex on the $\mathrm{Sb}$ surfaces, a robust zero-bias conductance peak emerges that does not split in a large distance when moving away from the vortex center on the Cs $2 \times 2$ surfaces, resembling the Majorana bound states arising from the superconducting Dirac surface states in $\mathrm{Bi}_{2} \mathrm{Te}_{3} / \mathrm{NbSe}_{2}$ heterostructures. Our findings establish $\mathrm{CsV}_{3} \mathrm{Sb}_{5}$ as a promising candidate for realizing exotic excitations at the confluence of nontrivial lattice geometry, topology and multiple electronic orders.
\end{abstract}

DOI: 10.1103/PhysRevX.11.031026

Subject Areas: Condensed Matter Physics

The kagome nets consisting of $3 d$ transition metal ions are emerging as a new frontier for exploring novel correlated and topological electronic states [1-10]. Thanks to its special lattice geometry, a kagome lattice naturally possesses Dirac cones and flat band with quenched kinetic energy [11,12], leading to interaction-driven topological many-body phenomena. Experimentally, 3D magnetic

\footnotetext{
${ }^{*}$ Corresponding author. lshan@ahu.edu.cn

Corresponding author. zywang2@ustc.edu.cn

"Corresponding author. chenxh@ustc.edu.cn

${ }^{\S}$ These authors contributed equally to this work

Published by the American Physical Society under the terms of the Creative Commons Attribution 4.0 International license. Further distribution of this work must maintain attribution to the author(s) and the published article's title, journal citation, and DOI.
}

Weyl semimetal [4,13-15] and 2D Chern insulator [9] phases have been realized in kagome lattices compounded with a net magnetization. On the other hand, depending on the electron filling level, various electronic states ranging from density waves to superconductivity have also been theoretically predicted [11,16-19]. However, the search for a layered kagome compound with a superconducting ground state remains a great challenge, leaving much of the related field unexplored.

Recently, a new family of layered kagome metals $A \mathrm{~V}_{3} \mathrm{Sb}_{5}(A=\mathrm{K}, \mathrm{Rb}, \mathrm{Cs})$ has been discovered with a superconducting ground state [20-23]. The normal state of these compounds has been theoretically identified to host a $Z_{2}$ topological invariant, with topological Dirac surface states near the Fermi energy (Dirac energy $E_{D} \sim 40 \mathrm{meV}$ ) and the calculated band structure is supported by angleresolved photoemission measurements [21]. In addition, these materials all exhibit a clear transport and magnetic anomaly at $T^{*} \sim 78-104 \mathrm{~K}$ due to the formation of a charge order [20-22], suggesting that the electronic interactions 

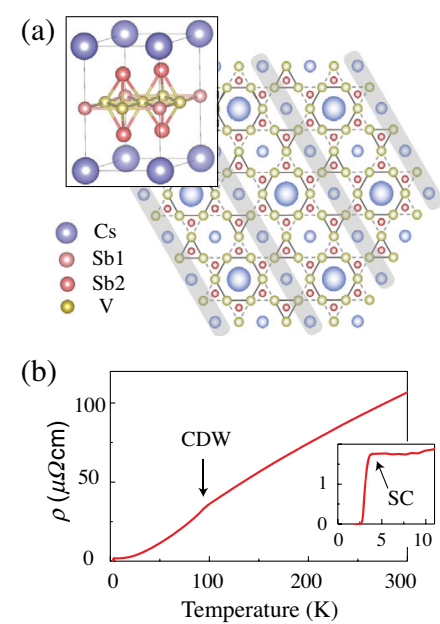
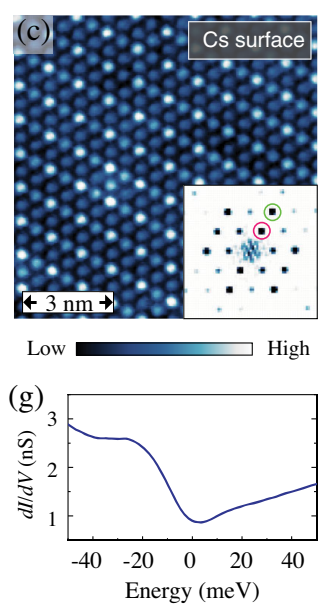
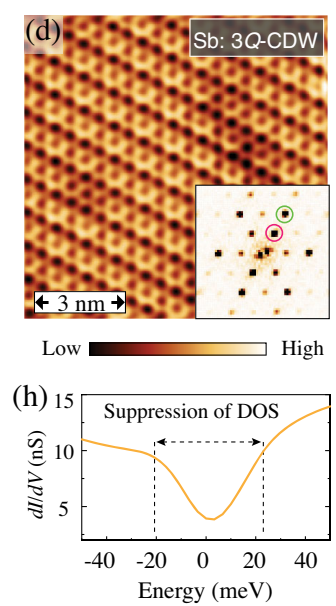
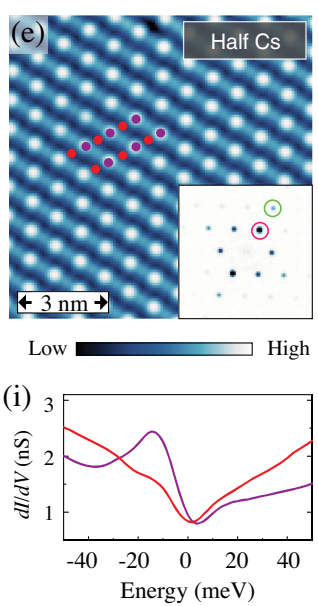
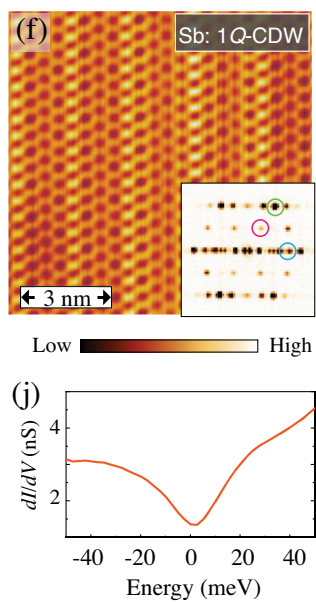

FIG. 1. (a) Crystal structure of $\mathrm{CsV}_{3} \mathrm{Sb}_{5}$ and the illustration of a top view of the lattice. The size of Cs atoms represents the CDW modulation. (b) Temperature-dependent resistivity of $\mathrm{CsV}_{3} \mathrm{Sb}_{5}$. A clear anomaly occurs around $94 \mathrm{~K}$, indicating the formation of charge order. The superconducting transition (marked as SC) that occurred at low temperature is shown in the inset. (c)-(e) Topographies of the $\mathrm{Cs}, \mathrm{Sb}$, and half-Cs surfaces, respectively. All of them show clear $3 Q-2 \times 2 \mathrm{CDW}$ patterns. (f) Topography obtained on $\mathrm{Sb}$ surfaces, showing additional $1 Q$ modulation. This $1 Q$ modulation is observed on large $\mathrm{Sb}$ surfaces with very few Cs atoms on the top, and it becomes weaker with more surrounding Cs atoms (SM 4 [31]). The insets display the Fourier transforms of these topographic images. The lattice peak is marked by green circles, the $3 Q$-CDW vector in red circles, while the $1 Q$-CDW vector is shown in blue. (g)-(j) Typical $d I / d V$ spectra for these surfaces. The purple and pink dots in (e) denote the locations where spectra are obtained for half-Cs surface. STM setup condition: (c) $V_{S}=-70 \mathrm{mV}, I_{t}=80 \mathrm{pA}$, (d) $V_{S}=-70 \mathrm{mV}, I_{t}=120 \mathrm{pA}$, (e) $V_{S}=-50 \mathrm{mV}, I_{t}=700 \mathrm{pA}$, (f) $V_{S}=100 \mathrm{mV}, I_{t}=150 \mathrm{pA},(\mathrm{g}) V_{S}=-60 \mathrm{mV}, I_{t}=150 \mathrm{pA}, V_{m}=3 \mathrm{mV}$, (h) $V_{S}=-50 \mathrm{mV}, I_{t}=450 \mathrm{pA}, V_{m}=4 \mathrm{mV}$, (i) $V_{S}=-60 \mathrm{mV}, I_{t}=250 \mathrm{pA}, V_{m}=3 \mathrm{mV}$, (j) $V_{S}=-50 \mathrm{mV}, I_{t}=130 \mathrm{pA}, V_{m}=2 \mathrm{mV}$.

could be at play in these compounds. This charge order has been revealed to be unconventional with a chiral anisotropy [24], giving rise to the observed anomalous Hall effect in the absence of long-range magnetic order [25,26]. When these $Z_{2}$ topological metals fall into a superconducting state, the natively proximitized superconducting topological surface states may provide a promising route to realize zero energy modes related to Majorana bound states (MBSs) [21,27], which are the key ingredients for topological quantum computation [28]. Furthermore, signatures of spin-triplet pairing and an edge supercurrent in $\mathrm{Nb} / \mathrm{K}_{1-X} \mathrm{~V}_{3} \mathrm{Sb}_{5}$ devices have been reported [29], suggesting a potential exotic superconducting state in these devices. Therefore, a spectroscopic probe of the electronic orders in $\mathrm{AV}_{3} \mathrm{Sb}_{5}$, particularly for the superconducting state, is highly desired.

Scanning tunneling microscopy (STM), owing to its high spatial topographic resolution and high energy spectroscopic resolution, is an ideal probe to investigate the real space charge ordering and novel quasiparticle excitations in the superconducting state. In this work, we use lowtemperature STM to study a kagome superconductor $\mathrm{CsV}_{3} \mathrm{Sb}_{5}$ with the highest $T_{C} \sim 3 \mathrm{~K}$ in the $A \mathrm{~V}_{3} \mathrm{Sb}_{5}$ family. We pinpoint a three-dimensional $2 \times 2 \times 2$ charge density wave (CDW) order in $\mathrm{CsV}_{3} \mathrm{Sb}_{5}$ with the opening of an energy gap of $\sim \pm 20 \mathrm{meV}$ at the Fermi level. Inside superconducting vortices on the ordered Cs surfaces, we observe a robust, nonsplit zero-bias conductance peak
(ZBCP) within a large distance away from the core center, whose full width at half maximum (FWHM) almost does not change. This $\mathrm{ZBCP}$ bears a remarkable resemblance to the MBSs that have been observed in the $\mathrm{Bi}_{2} \mathrm{Te}_{3} / \mathrm{NbSe}_{2}$ heterostructures [30].

Single crystals of $\mathrm{CsV}_{3} \mathrm{Sb}_{5}$ were grown via a selfflux growth method as described in earlier studies [20,21]. The crystals are first characterized by the x-ray diffraction [Supplemental Material note I (SM 1) [31]] and resistivity measurements. As shown in Fig. 1(b), a resistivity anomaly appears at $94 \mathrm{~K}$, similar to the previous report [21]. The onset of the superconductivity in our samples occurs around $3.5 \mathrm{~K}$, and zero resistivity can be achieved around $2.7 \mathrm{~K}$. The crystals were cold cleaved in situ at 30 or $80 \mathrm{~K}$, and then immediately transferred into the STM head. Chemically etched tungsten tips were used in all the measurements, after being checked on a clean $\mathrm{Au}$ (111) surface. STM measurements for the normal state and superconducting state are carried out at 5 and $0.4 \mathrm{~K}$, respectively. Spectroscopic data were acquired by the standard lock-in technique at a frequency of $987.5 \mathrm{~Hz}$.

$\mathrm{CsV}_{3} \mathrm{Sb}_{5}$ has a layered structure with the space group of $P 6 / \mathrm{mmm}$ and hexagonal lattice constant $a=5.5 \AA$ and $c=9.3 \AA$. It consists of a kagome vanadium net interpenetrated by a hexagonal net of Sb1 in between two honeycomb-structured Sb2 layers, and all sandwiched in between two hexagonal Cs layers [Fig. 1(a)]. Considering 
the chemical bonding between $\mathrm{V}, \mathrm{Sb} 2$, and $\mathrm{Cs}$, the main cleavage plane is in between the $\mathrm{Cs}$ and $\mathrm{Sb} 2$ layers, generating either $\mathrm{Cs}$ or $\mathrm{Sb}$ terminated surfaces. Atomically resolved STM topographies are shown in Figs. 1(c) and 1(d), with hexagonal and honeycomb lattice, respectively. In SM 2 [31], we show a joint area between these two types of lattices, with an atomic step edge where the upper regime shows hexagonal lattice while the lower shows honeycomb lattice. On the basis of the crystalline structure, we can identify them as Cs [Fig. 1(c)] and Sb [Fig. 1(d)] surfaces, respectively. One can see tridirectional $(3 Q) 2 \times 2$ superstructure in the real space topographies for both types of surfaces, with additional peaks at half of the Bragg reciprocal lattice vectors in the Fourier transforms (FTs), similar to the case of $\mathrm{KV}_{3} \mathrm{Sb}_{5}$ (see Ref. [24]). The observed modulation on both $\mathrm{Cs}$ and $\mathrm{Sb}$ surfaces suggests a $2 \times 2$ superlattice pattern in the underlying kagome V plane, as illustrated in Fig. 1(a), since the $3 d$ orbitals of $\mathrm{V}$ have a large contribution to the total electronic density of states (DOS) at low energies $[21,24]$. The $2 \times 2$ superlattice of $\mathrm{V}$ kagome lattice is plotted following an inverse Star of David structure as proposed by a recent density functional theory calculation [32]. The cleavage can also break the Cs atomic layer and result in a third type of surface with half the amount of the Cs atoms. In fact, this is the most commonly observed surface in our experiments. Figure 1(e) shows one of such surfaces, where half of the Cs chains are alternately missing, as illustrated by the gray lines in Fig. 1(a). Interestingly, we can still see clear charge modulation on all the remnant Cs chains [Fig. 1(e)] (more bias-dependent topographic images of half-Cs surface can be found in SM 3 [31]). We note that a unidirectional modulation with $4 a_{0}$ or $5 a_{0}$ lattice constant (1Q charge order; Ref. [33]), in addition to the $3 Q-2 \times 2$ order, can be observed on largely exposed $\mathrm{Sb}$ surfaces with only a few Cs atoms on the top [Fig. 1(f)], and becomes weak with more surrounding Cs atoms (SM 4 [31]). Thus, these two types of $3 Q$ and $1 Q$ charge orders may save similar total energy and can be influenced by strain $[34,35]$ or doping, especially near the surface.

Typically, in an electronic ordering phase, such as the classic Peierls-CDW state, one could observe a suppression of DOS near the Fermi level. We characterize the DOS by measuring the differential conductance $(d I / d V)$ and show the typical spectra far away from defects in Figs. 1(g)-1(j). There are several intriguing features in these spectra. Firstly, although a suppression of DOS near the $E_{F}$ can be observed for all these surfaces, this gaplike feature is clearer on the Sb surface, and we can deduce an energy scale of $\sim \pm 20 \mathrm{meV}$ from the change of slope in the spectral line shape. On the half-Cs surfaces, additional states appear at low energy (around $-12 \mathrm{meV}$ ), which exhibit spatial variations with the superlattice (SM 5 [31]) and change the apparent spectral line shape. Secondly, the suppression of DOS disappears above $94 \mathrm{~K}$, as shown in SM 6 [31], suggesting its close relationship with the formation of the superlattice. Thirdly, the additional modulation of the superlattice also leads to pronounced signatures in the spectroscopic images that reverses its intensity across the Fermi energy (SM 5 [31]), consistent with the CDW contribution to STM images observed in many CDW materials [36-38]. Thus, the observed superlattice modulation, the suppression of DOS near $E_{F}$, and the contrast inversion collectively support a CDW state in $\mathrm{CsV}_{3} \mathrm{Sb}_{5}$. In addition, we find that the minimum of the DOS is located slightly above $E_{F}$, similar to that observed in $\mathrm{NbSe}_{2}$ (see Ref. [34]). Although the driving mechanism of the CDW order remains unclear in $\mathrm{CsV}_{3} \mathrm{Sb}_{5}$, the particle-hole asymmetric gap indicates that it could not be prefect weak-coupled Fermi surface nesting, but may involve saddle points or strong electron-phonon coupling.

With the formation of $2 \times 2$ superlattice in the Cs layer, one would expect to see two types of topographies on the half-Cs surface, one with alternating bright and dark atoms in a chain while the other contains only dark Cs atoms without contrast changing [see Fig. 1(a) for an illustration where the dark-atom chains have been marked with gray lines], and the ratio between these two should be roughly $1: 1$. In spite of intensive search, only the former case has been observed on our cleaved samples. One possible reason for this deviation could be that the unit cell of $\mathrm{CsV}_{3} \mathrm{Sb}_{5}$ doubles along the $c$ axis in the CDW state, so that the Cs atoms on the topmost surface reflect the CDW pattern of the underlying $\mathrm{V}$ lattice which hosts a $\pi$-phase jump. A single-unit-cell surface step could offer a direct probe to check this picture. Figure 2(a) shows a topographic image including a single-unit-cell surface step edge [with the height of about $9 \AA$, Fig. 2(b)], which allows us to simultaneously view the CDW patterns of the upper and lower half-Cs surfaces side by side. A close-up view reveals that the CDW pattern exhibits a relative phase shift across the single-unit-cell step edge [Figs. 2(c) and 2(d); the topographic bright spots correspond to the bright sites in the CDW patterns]. To precisely determine the magnitude of the shift, a displacement analysis in reciprocal space is carried out (SM 7 [31]), which indicates that the phase shift is actually $\pi$. Measurements taken near the other two singleunit-cell steps are shown in SM 7 [31], and a similar $\pi$ phase jump has been observed. These observations further establish that the charge order in $\mathrm{CsV}_{3} \mathrm{Sb}_{5}$ is a threedimensional $2 \times 2 \times 2 \mathrm{CDW}$ state. The doubling of the unit cell along the $c$ direction may be related to the observed two-dome superconducting phase diagram under pressure [39].

We have performed spectroscopic imaging on the half-Cs surfaces. The FTs of the $d I / d V$ maps reveal clear quasiparticle interference (QPI) patterns [Figs. 2(e) and 2(f)] (more data can be found in SM 8 [31]). The white 

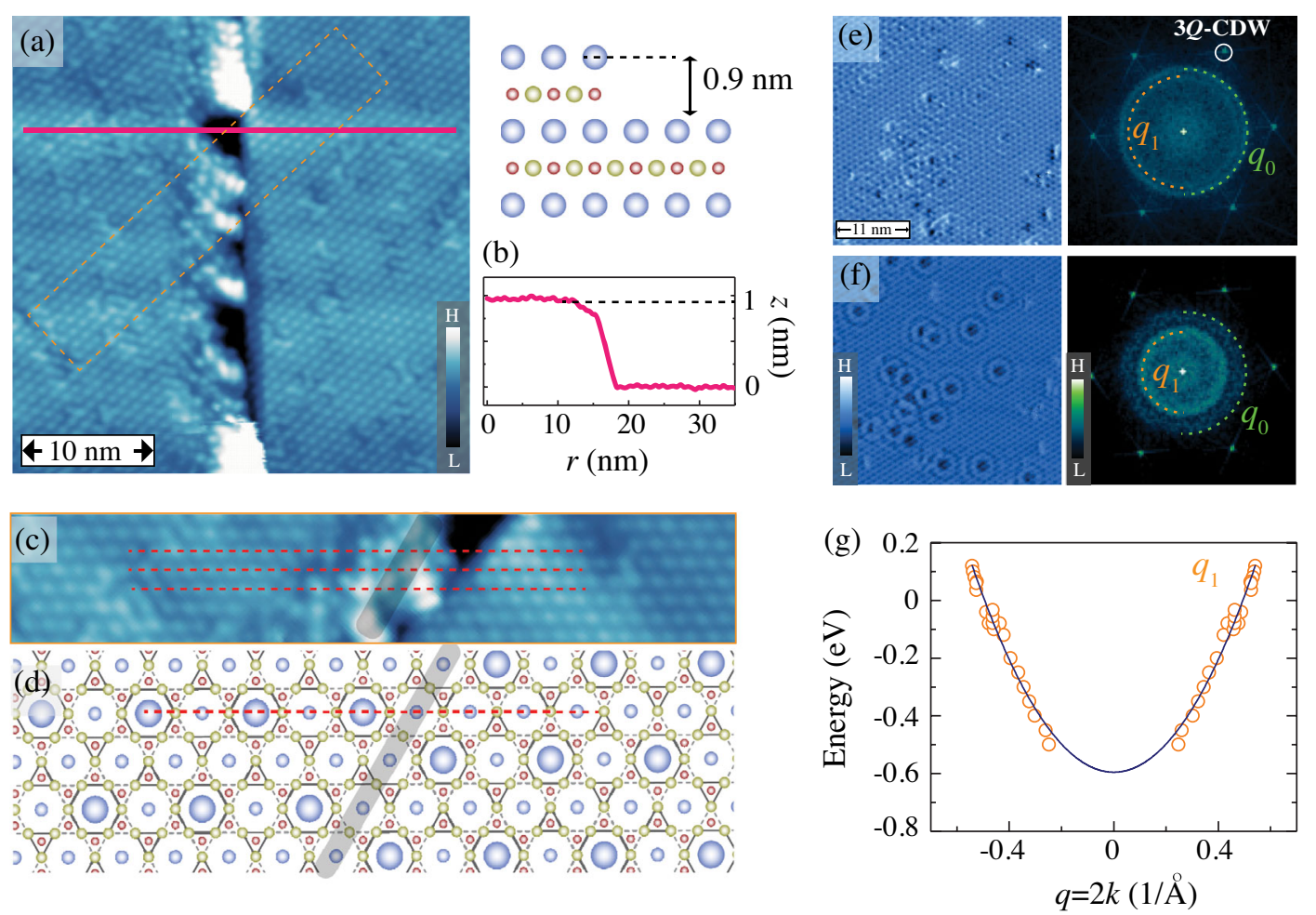

FIG. 2. (a) Topographic image of the half-Cs surface showing a single-unit-cell step. (b) Height profile obtained along the pink line shown in (a). (c) Close-up of the step edge. The dashed pink lines track the chains with CDW modulation on the upper side. A $\pi$-phase jump can be observed between the upper and lower sides. (d) Illustration of the CDW patterns near a single-unit-cell step. (e),(f) Normalized $d I / d V$ maps $(d I / d V(\boldsymbol{r}, \mathrm{eV}) /[I(\boldsymbol{r}, \mathrm{eV}) / V])$ and their FTs at -80 and $-300 \mathrm{mV}$, respectively. The white circle denotes one of the $3 Q$-CDW wave vectors. The clearly dispersing vector is marked as $q_{1}$. (g) The energy dispersion of $q_{1}$ extracted from line cuts of the FTs and a parabolic fit, $E=9.77 k^{2}-0.6$. STM setup condition: (a) $V_{S}=-100 \mathrm{mV}, I_{t}=80 \mathrm{pA}$, (e) $V_{S}=-80 \mathrm{mV}$, (f) $V_{S}=-300 \mathrm{mV}, I_{t}=450 \mathrm{pA}, V_{m}=4 \mathrm{mV}$.

circle in the FTs denotes the $3 Q$-CDW wave vector. The QPI data show two ringlike signals centered at the $\Gamma$ point. We find that the outer ring $\left(q_{0}\right)$ is almost nondispersing, which may potentially originate from Friedel oscillations $\left(2 k_{F}\right)$ or STM setpoint effect [40]. The inner one $\left(q_{1}\right)$ arises from the intrapocket scattering of the electron band at $\Gamma$, which has been assigned to the $p_{z}$ orbital of $\mathrm{Sb}$. The extracted dispersion of $q_{1}$, together with a parabolic fit, is displayed in Fig. $2(\mathrm{~g})$. The band bottom is estimated to be $-0.6 \mathrm{eV}$ from the fitting, consistent with the reported angle-resolved photoemission spectroscopy data [21].
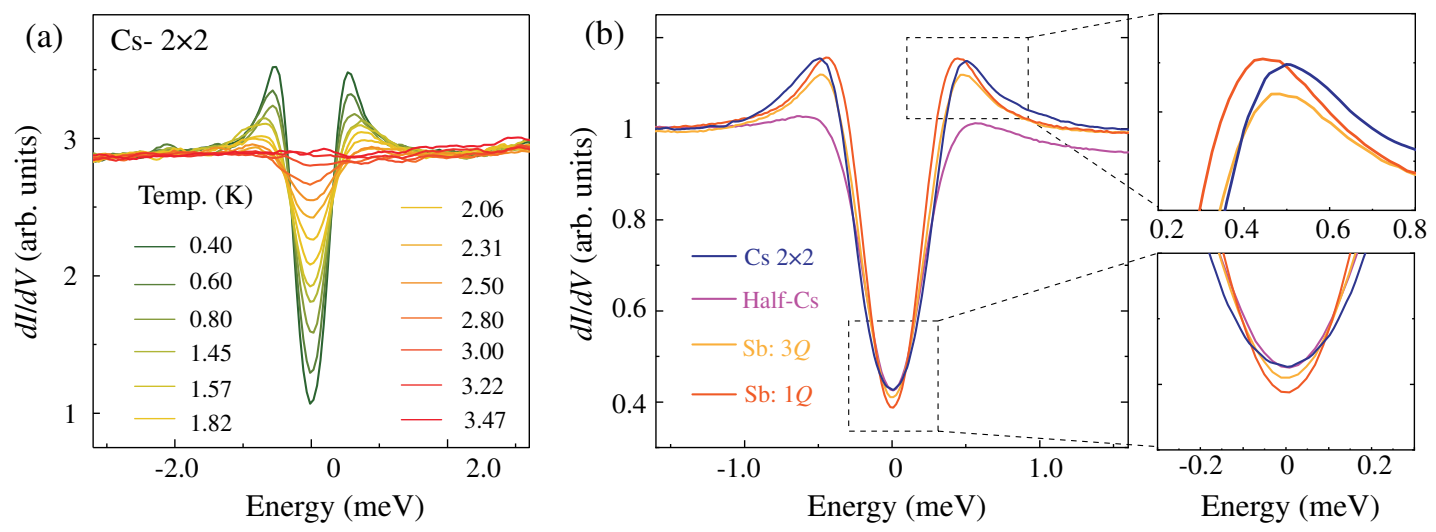

FIG. 3. (a) Temperature evolution of the superconducting gap. (b) Superconducting spectra obtained on different types of surfaces. The spectra of half-Cs, $\mathrm{Sb} 3 Q-2 \times 2$, and $\mathrm{Sb} 1 Q$ regions are obtained in one field of view with the same tip. STM setup condition: (a), (b) $V_{S}=-2 \mathrm{mV}, I_{t}=200 \mathrm{pA}, V_{m}=0.1 \mathrm{mV}$. 
(a)
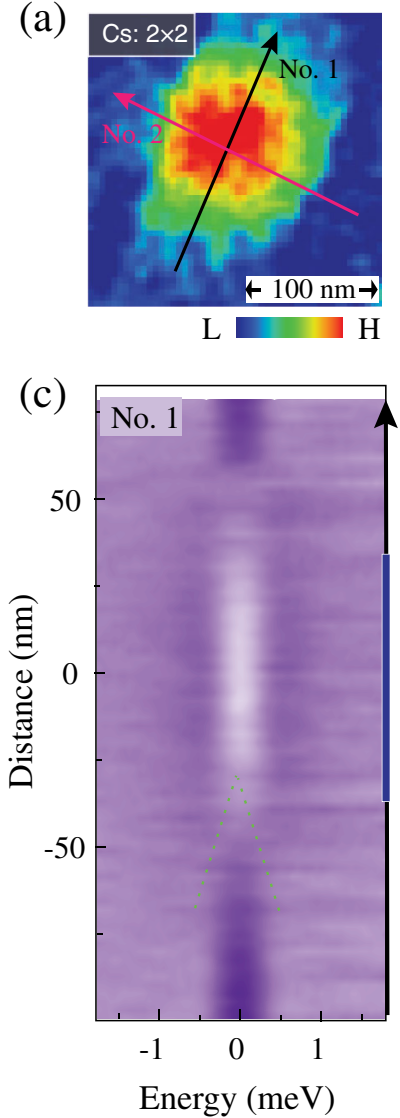

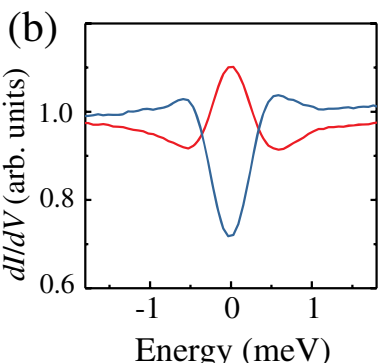

(d)

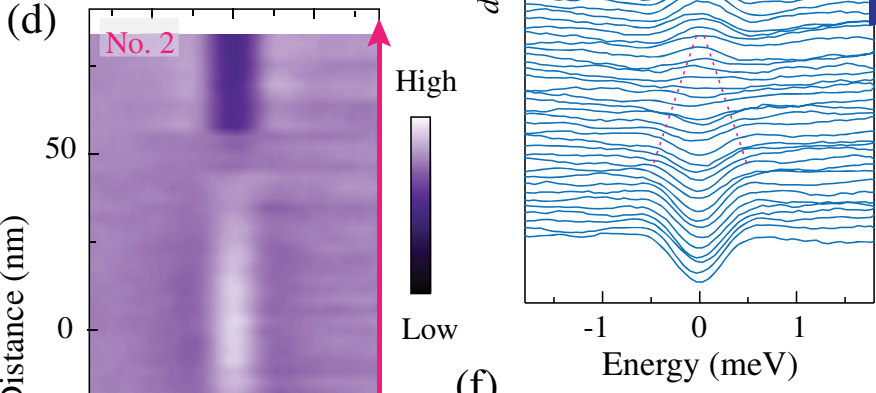

(f)

(e)

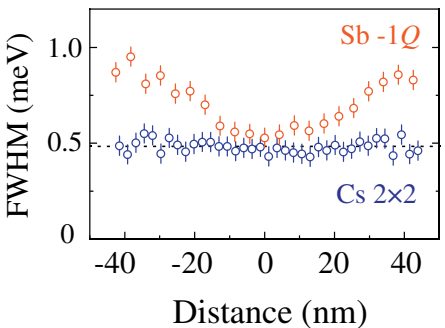

(g)

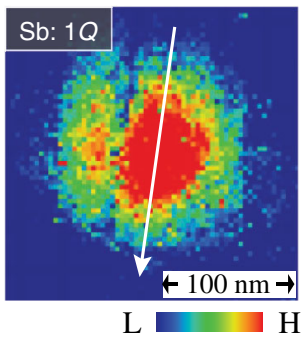

(h)

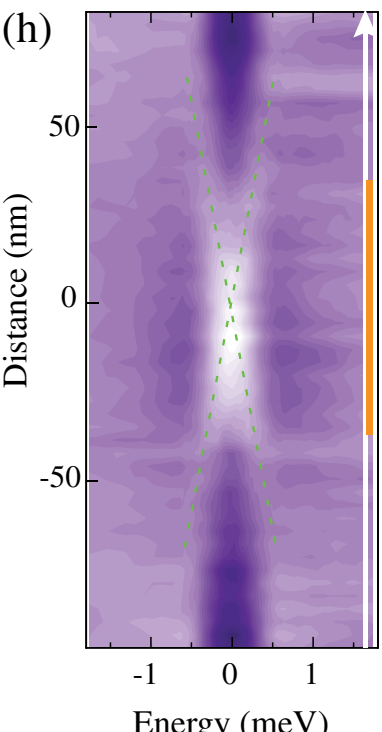

FIG. 4. (a) $d I / d V$ map showing a superconducting vortex on the Cs surface with $3 Q-2 \times 2$ CDW. (b) Tunneling spectra obtained in the vortex core (red) and outside the vortex (dark blue). (c),(d) Color map of the spatially resolved $d I / d V$ spectra across the vortex. The traces are marked by the dark and pink arrows as shown in (a). (e) The waterfall plot of (c), vertically offset for clarity. (f) Spatial dependence of FWHM of the ZBCP when moving away from the vortex core for Cs surface (blue) and Sb surface (orange). The error bar denotes the energy step used in the measurements. (g) $d I / d V$ map showing a vortex on the Sb surface with additional $1 Q$-CDW. (h) Spatial evolution of the $d I / d V$ spectra across the vortex. Dashed lines in (c)-(e) and (h) track the peak splitting. We note that the splitting of the peak is not clear near the starting point due to the limit of energy resolution; therefore, we track the visible splitting peaks near the vortex edge and use linear extrapolation to obtain the dashed lines (see Figs. S10 and S13 in Supplemental Material [31]). STM setup condition: (a) $V_{S}=-5 \mathrm{mV}, I_{t}=200 \mathrm{pA}, V_{m}=0.5 \mathrm{mV}$, (g) $V_{S}=-6 \mathrm{mV}, I_{t}=200 \mathrm{pA}, V_{m}=0.5 \mathrm{mV}$, (c),(d),(h) $V_{S}=-5 \mathrm{mV}, I_{t}=200 \mathrm{pA}, V_{m}=0.1 \mathrm{mV}$.

We now investigate the superconducting state of $\mathrm{CsV}_{3} \mathrm{Sb}_{5}$. At $0.4 \mathrm{~K}$, we observe a suppression of DOS at $E_{F}$ with symmetric coherence peaks forming at $\pm 0.55 \mathrm{meV}$ in the tunneling spectra [Fig. 3(a)], which we identify to be the superconducting gap. This gap is gradually suppressed at elevated temperatures and eventually disappears at the bulk $T_{c} \sim 3 \mathrm{~K}$. Interestingly, we find that the superconducting spectra obtained on different types of surfaces are slightly different. As summarized in Fig. 3(b), while the spectra acquired on the Cs $2 \times 2$ and $\mathrm{Sb}$ surfaces have more similar line shapes, the superconducting spectrum of the half-Cs surface shows clearly suppressed superconducting coherence peaks, indicating a larger scattering effect [41]. The overall evolution of the superconducting spectra has been checked on two samples. To gain a quantitative understanding on the superconducting order parameter, we fit the tunneling spectrum obtained on the Cs surface with the Dynes model [41], using isotropic $s$-wave gap, anisotropic $s$-wave, and two isotropic $s$-wave gap functions, respectively. The results can be found in SM 9 [31].

Next, we check the possible excitations inside a superconducting vortex. The vortices are directly imaged by mapping the conductance inside the superconducting gap with a magnetic field of $0.035 \mathrm{~T}$ perpendicular to the $a b$ plane. Based on the single magnetic flux quanta $2.07 \times 10^{-15} \mathrm{~Wb}$, one should see roughly one vortex in a $230 \times 230 \mathrm{~nm}^{2}$ field of view. Figure 4(a) shows such a map at zero energy with a single vortex on the Cs surface. We observe a clear ZBCP at the vortex core [Fig. 4(b)]. $d I / d V$ spectra acquired along two distinct paths [line 1 and line 2, denoted by the arrows in Fig. 4(a)] across the vortex reveal an intriguing evolution, which are shown in the false-color images of Figs. 4(c) and 4(d), respectively. 
When tracking the splitting peaks near the vortex core edge, we find that the start point of the splitting is located $\sim 35 \mathrm{~nm}$ away from the vortex core center (close-up of the splitting spectra is shown in SM 10 [31]), and the FWHM of the ZBCP bears little change in this range within our STM resolution $\sim 0.23 \mathrm{meV}$ (SM 11 [31]). This observation is in sharp contrast to the vortex core states in conventional $s$-wave superconductors, such as $\mathrm{NbSe}_{2}$ (see Ref. [42]), where an " $X$ "-type splitting has been widely observed, but is similar to the " $Y$ "-type splitting observed in 4-6 quintuple layer $\mathrm{Bi}_{2} \mathrm{Te}_{3} / \mathrm{NbSe}_{2}$ heterostructures [30] (a direct comparison has been shown in SM 10 [31]). We would like to emphasize that the ZBCP inside the vortex core is not induced by disorder for two reasons. First, at zero field, homogeneous superconducting spectra can be observed along the same trace of line 1 (SM 12 [31]), suggesting that the ZBCP indeed represents quasiparticle excitations inside a vortex. In fact, we never see an in-gap state that extends tens of nanometers at $0 \mathrm{~T}$ in our samples. Second, this $Y$-type splitting of ZBCP is repeatable once the vortex is located on the Cs surfaces (SM 13 [31]).

As we mentioned above, the largely exposed $\mathrm{Sb}$ surfaces exhibit additional $1 Q$ modulation, which is in contrast to the Cs surfaces that show only $3 Q-2 \times 2$ CDW. Interestingly, a distinct evolution of the vortex-core states is observed on the Sb surfaces. As shown in Figs. 4(g) and 4(h), the ZBCP inside the vortex core obtained on the Sb surface splits faster when moving away from the core center, exhibiting a clear $X$-type evolution that is more similar to the conventional case. As a result, the apparent width of the vortex-core states also shows remarkable spatial dependence, in contrast to that observed on the Cs surface [Fig. 4(f)]. For a better comparison, the spatial evolutions of vortex-core states observed on the Cs surface, half-Cs surface (with extra Cs atoms), and Sb surface, together with the atomic-resolution topographies, are presented in SM 14 [31].

The observation of distinct vortex-core states on different surfaces of $\mathrm{CsV}_{3} \mathrm{Sb}_{5}$ is not trivial. One possible explanation for the robust, finite-distance splitting $\mathrm{ZBCP}$ on the $3 Q-2 \times 2$ Cs surfaces is the presence of MBSs inside the vortex core. In the vortex of a conventional superconductor, discrete energy levels of Caroli-de GennesMatricon bound states (CBSs) appear at $E_{\mu}=\mu \Delta^{2} / E_{F}$, with $\mu$ the half-integer angular momentum number $(\mu= \pm 1 / 2 ; \pm 3 / 2 ; \pm 5 / 2 ; \ldots)$ [43]. The spatial distribution of specific CBS scales with its angular momentum number $\mu$, leading to a wave function that peaks at $r_{\mu} \sim|\mu| / k_{F}$ away from the vortex center. In most superconductors, the energy spacing of $\mu \Delta^{2} / E_{F}$ is too small to be discernible; thus a particle-hole symmetric ZBCP is experimentally observed at the core and it splits when moving right away from the vortex center, resulting in an $X$-type cross $[30,42,44,45]$. This is different from what we have found here. On the other hand, if Dirac surface states become superconducting, inside a vortex, quasiparticles gain an additional halfodd-integer contribution to the total angular momentum number owing to the intrinsic spin texture of Dirac fermions. Therefore, quasiparticles are integer quantized $(\mu=0, \pm 1, \pm 2 \ldots)$ and MBSs can be regarded as a special zero-energy CBS whose spin degree of freedom is eliminated. Theoretically, the MBS extends in real space with a length scale $\sim v_{F} / \Delta$ (similar to the superconducting coherence length) [46]. The presence of MBS could change the evolution of the quasiparticle spectra across the vortex, as seen in the cases of $\mathrm{Bi}_{2} \mathrm{Te}_{3} / \mathrm{NbSe}_{2}$ heterostructures [30] and $2 \mathrm{M}-\mathrm{WS}_{2}$ (see Ref. [47]). In $\mathrm{CsV}_{3} \mathrm{Sb}_{5}$, the superconducting gap could open on the bulk bands and topological surface states with tiny electron doping on the Cs surfaces, and they all contribute to the observed CBSs inside the vortex. The presence of MBSs is expected to enhance the conductance near zero energy within a length scale $\sim v_{F} / \Delta$ from the vortex center, resulting in the finitedistance ( $Y$-type) splitting of the ZBCP. By taking the slope of the calculated surface states of $\mathrm{CsV}_{3} \mathrm{Sb}_{5}(\sim 0.18 \mathrm{eV} \AA$ [21]) and experimentally extracted superconducting gap ( $\sim 0.4 \mathrm{meV}$, SM 9 [31]), if they exist, the MBSs would extend about $45 \mathrm{~nm}$ in real space, a value in good agreement with our experimental observation. Compared with iron-based superconductors $\mathrm{Fe}(\mathrm{Se}, \mathrm{Te})[48,49]$ and $(\mathrm{Li}$, $\mathrm{Fe}) \mathrm{OHFeSe}$ [50], the relatively large Fermi energies for both bulk bands and Dirac surface band in $\mathrm{CsV}_{3} \mathrm{Sb}_{5}$ impede the observation of discrete CBSs, making the case more similar to that of $\mathrm{Bi}_{2} \mathrm{Te}_{3} / \mathrm{NbSe}_{2}$ heterostructure.

Furthermore, the reported edge supercurrent in the $\mathrm{Nb} / \mathrm{K}_{1-X} \mathrm{~V}_{3} \mathrm{Sb}_{5}$ devices [29] and chiral anisotropy in the CDW states [24] indicate that the superconducting state in this family of compounds may also simultaneously break the time-reversal symmetry, making the situation more complex. A spin-triplet superconducting state with breaking time-reversal symmetry could also host MBSs inside the vortex core. To further reveal the superconductivity, possible future plans would include Bogoliubov QPI measurements to probe the superconducting gap functions $\Delta_{i}(k)$, and searching for possible edge states in the $d I / d V$ maps. Finally, we would like to emphasize that, although our STM data are consistent with the presence of MBSs inside the vortex core of $\mathrm{CsV}_{3} \mathrm{Sb}_{5}$, further investigations at lower temperature and on doped samples are necessary and more evidence is needed. Moreover, the distinct vortexcore states on the Cs and Sb surfaces, which may depend on local carrier doping [51] or different CDW modulations, would be an interesting topic for future theoretical study.

In summary, we have demonstrated a three-dimensional $2 \times 2 \times 2$ CDW state with an energy gap opening near the Fermi level in kagome superconductor $\mathrm{CsV}_{3} \mathrm{Sb}_{5}$. In the superconducting state, we find a ZBCP inside the vortex core that splits off at a large distance away from the vortex center on the Cs surfaces. This unusual splitting behavior, 
reminiscent of that observed in $\mathrm{Bi}_{2} \mathrm{Te}_{3} / \mathrm{NbSe}_{2}$, could possibly be due to the MBSs inside the vortex core. Our findings may have provided the first glimpse into possible Majorana modes in a kagome superconductor.

This work is supported by the National Key Research and Development Program of the Ministry of Science and Technology of China (No. 2017YFA0303001, No. 2017YFA0302904, and No. 2018YFA0305602), the National Natural Science Foundation of China (Grants No. 11888101, No. 12074002, and No. 11804379), the Strategic Priority Research Program of Chinese Academy of Sciences (Grant No. XDB25000000), Anhui Initiative in Quantum Information Technologies (Grant No. AHY160000), the Key Research Program of Frontier Sciences, CAS, China (QYZDYSSW-SLH021). Z. W. is supported by National Natural Science Foundation of China (No. 12074364), the Fundamental Research Funds for the Central Universities (WK3510000012), and USTC start-up fund. L.S. is supported by the Recruitment Program for Leading Talent Team of Anhui Province (2019-16).

[1] S. Nakatsuji, N. Kiyohara, and T. Higo, Large Anomalous Hall Effect in a Non-Collinear Antiferromagnet at Room Temperature, Nature (London) 527, 212 (2015).

[2] K. Kuroda et al., Evidence for Magnetic Weyl Fermions in a Correlated Metal, Nat. Mater. 16, 1090 (2017).

[3] L. Ye, M. Kang, J. Liu, F. von Cube, C. R. Wicker, T. Suzuki, C. Jozwiak, A. Bostwick, E. Rotenberg, D. C. Bell, L. Fu, R. Comin, and J. G. Checkelsky, Massive Dirac Fermions in a Ferromagnetic Kagome Metal, Nature (London) 555, 638 (2018).

[4] E. Liu et al., Giant Anomalous Hall Effect in a Ferromagnetic Kagome-Lattice Semimetal, Nat. Phys. 14, 1125 (2018).

[5] J.-X. Yin et al., Giant and Anisotropic Many-Body SpinOrbit Tunability in a Strongly Correlated Kagome Magnet, Nature (London) 562, 91 (2018).

[6] Q. Wang, Y. Xu, R. Lou, Z. Liu, M. Li, Y. Huang, D. Shen, H. Weng, S. Wang, and H. Lei, Large Intrinsic Anomalous Hall Effect in Half-Metallic Ferromagnet $\mathrm{Co}_{3} \mathrm{Sn}_{2} \mathrm{~S}_{2}$ with Magnetic Weyl Fermions, Nat. Commun. 9, 3681 (2018).

[7] Z. Lin, J.-H. Choi, Q. Zhang, W. Qin, S. Yi, P. Wang, L. Li, Y. Wang, H. Zhang, Z. Sun, L. Wei, S. Zhang, T. Guo, Q. Lu, J.-H. Cho, C. Zeng, and Z. Zhang, Flatbands and Emergent Ferromagnetic Ordering in $\mathrm{Fe}_{3} \mathrm{Sn}_{2}$ Kagome Lattices, Phys. Rev. Lett. 121, 096401 (2018).

[8] M. Kang et al., Dirac Fermions and Flat Bands in the Ideal Kagome Metal FeSn, Nat. Mater. 19, 163 (2020).

[9] J.-X. Yin et al., Quantum-Limit Chern Topological Magnetism in $\mathrm{TbMn}_{6} \mathrm{Sn}_{6}$, Nature (London) 583, 533 (2020).

[10] Y. Xing et al., Localized Spin-Orbit Polaron in Magnetic Weyl Semimetal $\mathrm{Co}_{3} \mathrm{Sn}_{2} \mathrm{~S}_{2}$, Nat. Commun. 11, 5613 (2020).

[11] H. M. Guo and M. Franz, Topological Insulator on the Kagome Lattice, Phys. Rev. B 80, 113102 (2009).

[12] I. I. Mazin, H. O. Jeschke, F. Lechermann, H. Lee, M. Fink, R. Thomale, and R. Valentí, Theoretical Prediction of a
Strongly Correlated Dirac Metal, Nat. Commun. 5, 4261 (2014).

[13] D. F. Liu, A. J. Liang, E. K. Liu, Q. N. Xu, Y. W. Li, C. Chen, D. Pei, W. J. Shi, S. K. Mo, P. Dudin, T. Kim, C. Cacho, G. Li, Y. Sun, L. X. Yang, Z. K. Liu, S. S. P. Parkin, C. Felser, and Y. L. Chen, Magnetic Weyl Semimetal Phase in a Kagomé Crystal, Science 365, 1282 (2019).

[14] I. Belopolski et al., Discovery of Topological Weyl Fermion Lines and Drumhead Surface States in a Room Temperature Magnet, Science 365, 1278 (2019).

[15] N. Morali, R. Batabyal, P. K. Nag, E. Liu, Q. Xu, Y. Sun, B. Yan, C. Felser, N. Avraham, and H. Beidenkopf, Fermi-Arc Diversity on Surface Terminations of the Magnetic Weyl Semimetal $\mathrm{Co}_{3} \mathrm{Sn}_{2} \mathrm{~S}_{2}$, Science 365, 1286 (2019).

[16] J. Wen, A. Rüegg, C. C. J. Wang, and G. A. Fiete, Interaction-Driven Topological Insulators on the Kagome and the Decorated Honeycomb Lattices, Phys. Rev. B 82, 075125 (2010).

[17] W.-H. Ko, P. A. Lee, and X.-G. Wen, Doped Kagome System as Exotic Superconductor, Phys. Rev. B 79, 214502 (2009).

[18] M. L. Kiesel, C. Platt, and R. Thomale, Unconventional Fermi Surface Instabilities in the Kagome Hubbard Model, Phys. Rev. Lett. 110, 126405 (2013).

[19] S.-L. Yu and J.-X. Li, Chiral Superconducting Phase and Chiral Spin-Density-Wave Phase in a Hubbard Model on the Kagome Lattice, Phys. Rev. B 85, 144402 (2012).

[20] B. R. Ortiz, L. C. Gomes, J. R. Morey, M. Winiarski, M. Bordelon, J. S. Mangum, I. W. H. Oswald, J. A. Rodriguez-Rivera, J. R. Neilson, S. D. Wilson, E. Ertekin, T. M. McQueen, and E. S. Toberer, New Kagome Prototype Materials: Discovery of $\mathrm{KV}_{3} \mathrm{Sb}_{5}, \mathrm{RbV}_{3} \mathrm{Sb}_{5}$, and $\mathrm{CsV}_{3} \mathrm{Sb}_{5}$, Phys. Rev. Mater. 3, 094407 (2019).

[21] B. R. Ortiz, S. M. L. Teicher, Y. Hu, J. L. Zuo, P. M. Sarte, E. C. Schueller, A. M. M. Abeykoon, M. J. Krogstad, S. Rosenkranz, R. Osborn, R. Seshadri, L. Balents, J. He, and S. D. Wilson, $\mathrm{CsV}_{3} \mathrm{Sb}_{5}: A \mathrm{Z}_{2}$ Topological Kagome Metal with a Superconducting Ground State, Phys. Rev. Lett. 125, 247002 (2020).

[22] B. R. Ortiz, P. M. Sarte, E. M. Kenney, M. J. Graf, S. M. L. Teicher, R. Seshadri, and S. D. Wilson, Superconductivity in the $\mathrm{Z}_{2}$ Kagome Metal $\mathrm{KV}_{3} \mathrm{Sb}_{5}$, Phys. Rev. Mater. 5, 034801 (2021).

[23] Q. Yin, Z. Tu, C. Gong, Y. Fu, S. Yan, and H. Lei, Superconductivity and Normal-State Properties of Kagome Metal $\mathrm{RbV}_{3} \mathrm{Sb}_{5}$ Single Crystals, Chin. Phys. Lett. 38, 037403 (2021).

[24] Y.-X. Jiang et al., Unconventional Chiral Charge Order in Kagome Superconductor $\mathrm{KV}_{3} \mathrm{Sb}_{5}$, Nat. Mater. (2021), https://doi.org/10.1038/s41563-021-01034-y.

[25] S.-Y. Yang, Y. Wang, B. R. Ortiz, D. Liu, J. Gayles, E. Derunova, R. Gonzalez-Hernandez, L. Šmejkal, Y. Chen, S. S. P. Parkin, S. D. Wilson, E. S. Toberer, T. McQueen, and M. N. Ali, Giant, Unconventional Anomalous Hall Effect in the Metallic Frustrated Magnet Candidate, $\mathrm{KV}_{3} \mathrm{Sb}_{5}$, Sci. Adv. 6, eabb6003 (2020).

[26] F. H. Yu, T. Wu, Z. Y. Wang, B. Lei, W. Z. Zhuo, J. J. Ying, and X. H. Chen, Concurrence of Anomalous Hall Effect and Charge Density Wave in a Superconducting Topological Kagome Metal, arXiv:2102.10987. 
[27] L. Fu and C. L. Kane, Superconducting Proximity Effect and Majorana Fermions at the Surface of a Topological Insulator, Phys. Rev. Lett. 100, 096407 (2008).

[28] A. Kitaev, Fault-Tolerant Quantum Computation by Anyons, Ann. Phys. (Amsterdam) 303, 2 (2003).

[29] Y. Wang, S. Yang, P. K. Sivakumar, B. R. Ortiz, S. M. L. Teicher, H. Wu, A. K. Srivastava, C. Garg, D. Liu, S. S. P. Parkin, E. S. Toberer, T. McQueen, S. D. Wilson, and M. N. Ali, Proximity-Induced Spin-Triplet Superconductivity and Edge Supercurrent in the Topological Kagome Metal, $\mathrm{K}_{1-x} \mathrm{~V}_{3} \mathrm{Sb}_{5}$, arXiv:2012.05898.

[30] J.-P. Xu, M.-X. Wang, Z. L. Liu, J.-F. Ge, X. Yang, C. Liu, Z. A. Xu, D. Guan, C. L. Gao, D. Qian, Y. Liu, Q.-H. Wang, F.-C. Zhang, Q.-K. Xue, and J.-F. Jia, Experimental Detection of a Majorana Mode in the Core of a Magnetic Vortex Inside a Topological Insulator-Superconductor $\mathrm{Bi}_{2} \mathrm{Te}_{3} / \mathrm{NbSe}_{2}$ Heterostructure, Phys. Rev. Lett. 114, 017001 (2015).

[31] See Supplemental Material athttp://link.aps.org/ supplemental/10.1103/PhysRevX.11.031026 for more topographies and tunneling spectra of the CDW state, displacement analysis near a single-unit-cell step, fitting of the superconducting spectra and more vortex data on different surfaces.

[32] H. Tan, Y. Liu, Z. Wang, and B. Yan, Charge Density Waves and Electronic Properties of Superconducting Kagome Metals, arXiv:2103.06325.

[33] H. Zhao, H. Li, B. R. Ortiz, S. M. L. Teicher, T. Park, M. Ye, Z. Wang, L. Balents, S. D. Wilson, and I. Zeljkovic, Cascade of Correlated Electron States in a Kagome Superconductor $\mathrm{CsV}_{3} \mathrm{Sb}_{5}$, arXiv:2103.03118.

[34] A. Soumyanarayanan, M. M. Yee, Y. He, J. van Wezel, D. J. Rahn, K. Rossnagel, E. W. Hudson, M. R. Norman, and J. E. Hoffman, Quantum Phase Transition from Triangular to Stripe Charge Order in $\mathrm{NbSe}_{2}$, Proc. Natl. Acad. Sci. U.S.A. 110, 1623 (2013).

[35] S. Gao, F. Flicker, R. Sankar, H. Zhao, Z. Ren, B. Rachmilowitz, S. Balachandar, F. Chou, K. S. Burch, Z. Wang, J. van Wezel, and I. Zeljkovic, Atomic-Scale Strain Manipulation of a Charge Density Wave, Proc. Natl. Acad. Sci. U.S.A. 115, 6986 (2018).

[36] P. Mallet, K. M. Zimmermann, P. Chevalier, J. Marcus, J. Y. Veuillen, and J. M. G. Rodriguez, Contrast Reversal of the Charge Density Wave STM Image in Purple Potassium Molybdenum Bronze $\mathrm{K}_{0.9} \mathrm{Mo}_{6} \mathrm{O}_{17}$, Phys. Rev. B 60, 2122 (1999).

[37] D. Stoltz, M. Bielmann, M. Bovet, L. Schlapbach, and H. Berger, Tunneling Evidence for Spatial Location of the Charge-Density-Wave Induced Band Splitting in $1 T-\mathrm{TaSe}_{2}$, Phys. Rev. B 76, 073410 (2007).

[38] M. Spera, A. Scarfato, Á. Pásztor, E. Giannini, D. R. Bowler, and C. Renner, Insight into the Charge Density Wave Gap from Contrast Inversion in Topographic STM Images, Phys. Rev. Lett. 125, 267603 (2020).
[39] K. Y. Chen, N. N. Wang, Q. W. Yin, Z. J. Tu, C. S. Gong, J. P. Sun, H. C. Lei, Y. Uwatoko, and J.-G. Cheng, Double Superconducting Dome and Triple Enhancement of Tc in the Kagome Superconductor $\mathrm{CsV}_{3} \mathrm{Sb}_{5}$ under High Pressure, arXiv:2102.09328 [Phys. Rev. Lett. (to be published)].

[40] A. Macdonald, Y.-S. Tremblay-Johnston, S. Grothe, S. Chi, P. Dosanjh, S. Johnston, and S. A. Burke, Dispersing Artifacts in FT-STS: A Comparison of Set Point Effects across Acquisition Modes, Nanotechnology 27, 414004 (2016).

[41] R. C. Dynes, J. P. Garno, G. B. Hertel, and T. P. Orlando, Tunneling Study of Superconductivity Near the MetalInsulator Transition, Phys. Rev. Lett. 53, 2437 (1984).

[42] H. F. Hess, R. B. Robinson, and J. V. Waszczak, VortexCore Structure Observed with a Scanning Tunneling Microscope, Phys. Rev. Lett. 64, 2711 (1990).

[43] L. Kramer and W. Pesch, Core Structure and Low-Energy Spectrum of Isolated Vortex Lines in Clean Superconductors at $T \ll T_{c}$, Z. Phys. 269, 59 (1974).

[44] F. Gygi and M. Schlüter, Self-Consistent Electronic Structure of a Vortex Line in a Type-II superconductor, Phys. Rev. B 43, 7609 (1991).

[45] H. F. Hess, R. B. Robinson, R. C. Dynes, J. M. Valles, and J. V. Waszczak, Scanning-Tunneling-Microscope Observation of the Abrikosov Flux Lattice and the Density of States near and inside a Fluxoid, Phys. Rev. Lett. 62, 214 (1989).

[46] C.-K. Chiu, M. J. Gilbert, and T. L. Hughes, Vortex Lines in Topological Insulator-Superconductor Heterostructures, Phys. Rev. B 84, 144507 (2011).

[47] Y. Yuan, J. Pan, X. Wang, Y. Fang, C. Song, L. Wang, K. He, X. Ma, H. Zhang, F. Huang, W. Li, and Q.-K. Xue, Evidence of Anisotropic Majorana Bound States in $2 M-\mathrm{WS}_{2}$, Nat. Phys. 15, 1046 (2019).

[48] D. Wang, L. Kong, P. Fan, H. Chen, S. Zhu, W. Liu, L. Cao, Y. Sun, S. Du, J. Schneeloch, R. Zhong, G. Gu, L. Fu, H. Ding, and H.-J. Gao, Evidence for Majorana Bound States in an Iron-Based Superconductor, Science 362, 333 (2018).

[49] L. Kong, S. Zhu, M. Papaj, H. Chen, L. Cao, H. Isobe, Y. Xing, W. Liu, D. Wang, P. Fan, Y. Sun, S. Du, J. Schneeloch, R. Zhong, G. Gu, L. Fu, H.-J. Gao, and H. Ding, Half-Integer Level Shift of Vortex Bound States in an Iron-Based Superconductor, Nat. Phys. 15, 1181 (2019).

[50] Q. Liu, C. Chen, T. Zhang, R. Peng, Y.-J. Yan, C.-H.-P. Wen, X. Lou, Y.-L. Huang, J.-P. Tian, X.-L. Dong, G.-W. Wang, W.-C. Bao, Q.-H. Wang, Z.-P. Yin, Z.-X. Zhao, and D.-L. Feng, Robust and Clean Majorana Zero Mode in the Vortex Core of High-Temperature Superconductor ( $\left.\mathrm{Li}_{0.84} \mathrm{Fe}_{0.16}\right)$ OHFeSe, Phys. Rev. X 8, 041056 (2018).

[51] P. Hosur, P. Ghaemi, R. S. K. Mong, and A. Vishwanath, Majorana Modes at the Ends of Superconductor Vortices in Doped Topological Insulators, Phys. Rev. Lett. 107, 097001 (2011). 